\title{
Pilot study of a combination of S-1 and paclitaxel for patients with peritoneal metastasis from gastric cancer
}

\author{
Shigeyuki Tamura, Hirofumi Miki, Kaoru Okada, Atsushi Takeno, Kumiko Uji, Atsuko Yoshida, Rei Suzuki, \\ Shin Nakahira, Chiyomi Egawa, Ken Nakata, Shu Okamura, Keishi Sugimoto, and Yuichi Takatsuka
}

Department of Surgery, Kansai Rosai Hospital, 3-1-69 Inabaso, Amagasaki, Hyogo 660-8511, Japan

\begin{abstract}
Background. This pilot study was carried out to evaluate the efficacy of chemotherapy for patients with peritoneal dissemination from gastric cancer or positive lavage cytology diagnosed by staging laparoscopy.

Methods. Sixteen patients were enrolled. Paclitaxel was administered at $120 \mathrm{mg} / \mathrm{m}^{2}$ on day 1 and $S-1$ was administered orally at $80 \mathrm{mg} / \mathrm{m}^{2}$ for 14 consecutive days, followed by a 1-week rest, as one course. After five courses of this therapy, the primary gastric tumors were evaluated and second-look laparoscopy was performed for patients showing partial response or stable disease with clinical benefit.

Results. Partial response or stable disease with clinical benefit was confirmed in seven and five patients, respectively, and these patients underwent second-look laparoscopy. No viable cancer cells were detected on cytopathological investigation during second-look laparoscopy in 9 patients who underwent surgical treatment. The intent-to-treat response rate for gastric tumor was $44 \%$ and the rate of disappearance of peritoneal metastasis was $38 \%$ (6 cases) at surgery. The median survival time was 555 days. Leucopenia of grade 3 and neutropenia of grade 3 were recognized in two and three patients, respectively.

Conclusion. This chemotherapy regimen may be an acceptable option for patients with peritoneal dissemination. We plan to study this regimen further in gastric cancer patients with peritoneal dissemination.
\end{abstract}

Key words Advanced gastric cancer - Peritoneal dissemination $\cdot$ Combination chemotherapy $\cdot$ Paclitaxel $\cdot \mathrm{S}-1$

\section{Introduction}

Advances in early diagnosis and surgical techniques have improved the outcome of treatment for gastric cancer. However, gastric cancer patients with peritoneal

Offprint requests to: S. Tamura

Received: October 6, 2009 / Accepted: February 10, 2010 dissemination still have a poor prognosis [1]; most of these patients die within 6 months of diagnosis, while the 5-year survival rate is nil $[2,3]$.

The recent introduction of an oral drug, S- 1 , has increased the overall response rates (ORRs) to $44 \%$ and $49 \%$ and median survival time (MST) to 8 months in phase II studies [4, 5].

Several reports have demonstrated that S-1 was effective for undifferentiated histological types, such as poorly differentiated adenocarcinoma and signet-ring cell carcinoma, which are relevant to peritoneal dissemination [6]. S-1 has been reported to be effective in prolonging the survival of gastric cancer patients with peritoneal dissemination [4-7].

Moreover, S-1 in combination with other anticancer drugs such as cisplatin (CDDP), taxanes, and irinotecan (CPT-11) increased ORRs and prolonged MST [8-11].

Paclitaxel is a cytotoxic antineoplastic agent that causes excessive polymerization of tubulin and microtubule dysfunction, resulting in tumor cell death [12]. Kobayashi et al. [13] have demonstrated that paclitaxel is a promising drug for the treatment of malignant ascites in patients with gastric cancer: the concentration of paclitaxel in ascites was maintained within the optimal level for the treatment of cancer cells for up to $72 \mathrm{~h}$ after intravenous administration.

Recent phase II studies of systemic chemotherapy with S-1 plus taxanes have demonstrated strong anticancer effects and a good MST in the treatment of advanced gastric cancer $[9,10,14]$. However, the efficacy of systemic chemotherapy for peritoneal dissemination from gastric cancer has been unclear, because peritoneal metastases have not been defined as measurable lesions in conventional phase II studies. Therefore, few reports describing the efficacy of chemotherapy for these lesions are available.

In this pilot study, we planned therapeutic strategies to observe the effect of chemotherapy on peritoneal metastasis by second-look laparoscopy for patients 
showing some clinical improvement following this combined chemotherapy and to perform surgical resection for patients in whom peritoneal metastasis had disappeared. In this study, patients with peritoneal dissemination or positive lavage cytology were defined as patients with peritoneal metastasis, because patients with positive lavage cytology show a poor prognosis even when curative resection for gastric cancer is performed - the same as that shown for patients with peritoneal dissemination $[15,16]$.

This study examined the safety and efficacy of chemotherapy for peritoneal dissemination as a prospective pilot study.

\section{Patients and methods}

\section{Patients}

Patients were enrolled in this study between June 2003 and August 2007. Eligible patients had histologically proven peritoneal dissemination (macroscopic peritoneal metastasis) or cytologically confirmed positive peritoneal lavage cytology (microscopic peritoneal metastasis) from gastric adenocarcinoma on staging laparoscopy. Patients with distant metastasis such as liver metastasis and/or bulky lymph node metastasis adjacent to the common hepatic artery, left gastric artery, and/or celiac axis were excluded, because such patients should receive chemotherapy as a first line of treatment regardless of peritoneal metastasis.

Other inclusion criteria were as follows: age 20-75 years; Eastern Clinical Oncology Group performance status of $0-1$; hemoglobin more than $10.0 \mathrm{~g} / \mathrm{dl}$; white blood cell count $4000 \mathrm{~mm}^{3}$ or more; neutrophil count $2000 \mathrm{~mm}^{3}$ or more; platelet count $100000 \mathrm{~mm}^{3}$ or more; aspartate aminotranferase (AST) and alanine amino- transferase (ALT) less than $100 \mathrm{U} / \mathrm{l}$; serum bilirubin $1.5 \mathrm{mg} / \mathrm{dl}$ or less; serum creatinine $1.2 \mathrm{mg} / \mathrm{dl}$ or less (our upper institutional limit); normal electrocardiogram; and the absence of other severe medical conditions or synchronous malignancy.

Exclusion criteria were as follows: infection or suspected infection, with fever; congestive heart failure; uncontrolled angina pectoris or arrhythmia; uncontrolled diabetes or hypertension; interstitial pneumonia or lung fibrosis; symptomatic brain metastasis; and pregnancy. Patients with a history of prior chemotherapy were also excluded.

Informed consent was obtained from each patient before study entry.

\section{Laparoscopy}

Laparoscopy was performed under general anesthesia as an independent procedure by the method we reported previously [17]. After close examination of the peritoneal cavity, peritoneal lavage cytology was performed in the Douglas pouch and left subphrenic space. If there were any suspected metastatic lesions, biopsy of the lesions was performed for histological examination.

After the chemotherapy was completed, conventional examinations were carried out to assess the clinical response; the assessment was done according to the Japanese classification of gastric carcinoma, 2nd English edition [18] and the Japanese clinical criteria for accessment of response to nonsurgical treatment of gastric cancer [19]. If the treatment was judged to have induced a partial response or minimal response with clinical benefit, such as an increase in appetite and amelioration of abdominal symptoms, second-look laparoscopy was performed to assess the effectiveness of the treatment against peritoneal metastasis (Fig. 1).

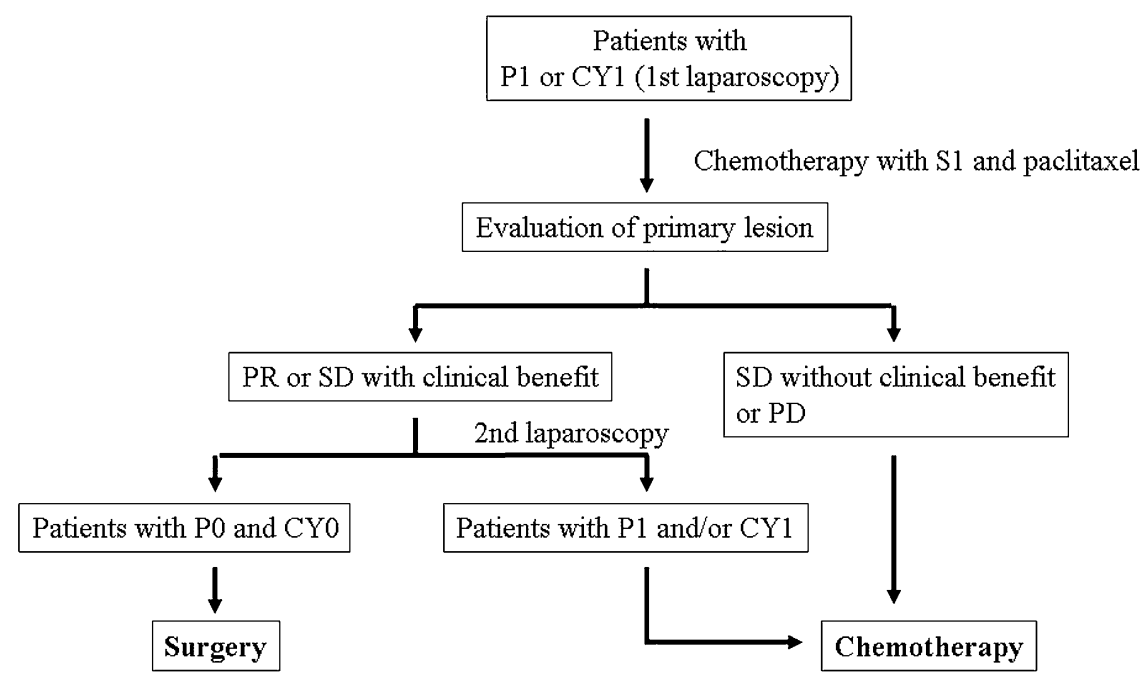

Fig. 1. Treatment schedule. $P$, peritoneal dissemination; $\quad C Y$, intra-abdominal lavage cytology; $P R$, partial response; $S D$, stable disease; $P D$, progressive disease; clinical benefit, clinical improvement such as increased appetite or amelioration of abdominal symptoms; $P 1$, peritoneal metastasis; $P 0$, no peritoneal metastasis; $C Y 1$, cancer cells on peritoneal cytology; $C Y 0$, benign/intermediate cells on peritoneal cytology 


\section{Treatment schedules}

S-1 was administered orally at $80 \mathrm{mg} / \mathrm{m}^{2}$ for 14 consecutive days, followed by 7 days' rest, as one course. Paclitaxel was administered intravenously, at a dose of $120 \mathrm{mg} / \mathrm{m}^{2}$ in $500 \mathrm{ml}$ of normal saline, given over a 30 -min period, on day 1 , following premedication with dexamethasone $8 \mathrm{mg}$ and ranitidine hydrochloride $50 \mathrm{mg}$, given as a intravenous infusion $60 \mathrm{~min}$ prior to paclitaxel administration.

This 3-week treatment cycle was repeated five times.

\section{Objective responses and toxicities}

The extent of disease was evaluated by physical examination, chest X-ray, computed tomography (CT), upper gastrointestinal series (UGI), and endoscopic examination of the upper gastrointestinal tract before entry into the study and after treatment. Blood cell counts and hepatic and renal function tests were conducted at least once every 3 weeks during the chemotherapy courses.

As the clinical response could not be objectively evaluated according to the response evaluation criteria in solid tumors (RECIST), as indicated above, the responses of the primary tumors were evaluated after five cycles of this treatment based on roentgenographic and endoscopic findings, according to the response criteria of the Japanese Gastric Cancer Association [19]. In this study, complete response of peritoneal dissemination was defined as confirmation of both the disappearance of peritoneal dissemination and negative lavage cytology at both second-look laparoscopy and surgery.

The histological effects on the primary tumor were classified into three categories, and grade 1 was further divided into two subcategories according to the Japanese classification of gastric carcinoma. Namely, grade 1, less than two-thirds tumor shrinkage (grade 1a, less than one-third tumor shrinkage; grade $1 \mathrm{~b}$, between onethird and two-thirds tumor shrinkage); grade 2, more than two-thirds tumor shrinkage; and grade 3, complete regression.

The National Cancer Institute Common Toxicity Criteria (NCI-CTC) version 2 were applied to evaluate adverse drug reactions.

\section{Statistical analysis}

Overall survival (OS) was calculated from the first day of the chemotherapy to the date of death from any cause or last follow-up visit in 2009. Analysis of the OS curve was performed using the Kaplan-Meier method. All statistical analyses were performed using SPSS software (SPSS, Chicago, IL, USA).

\section{Results}

\section{Patient characteristics}

Sixteen patients were entered into this study. Ten patients had peritoneal dissemination and 13 had positive lavage cytology proved by staging laparoscopy; 6 had only positive lavage cytology without peritoneal dissemination. The median age was 66 years (range, 48-73 years). Twelve patients were male and four were female. Patient characteristics are summarized in Table 1. All patients had nonmeasurable lesions, because patients with measurable lesions had received chemotherapy without staging laparoscopy and had been excluded. Histologically, one patient had intestinal-type adenocarcinoma, and 15 had diffuse-type adenocarcinoma.

\section{Efficacy and second-look laparoscopy}

Table 2 summarizes the clinical courses of all the patients. One of the 16 patients was not evaluable for response because gastrectomy was performed for acute bleeding from the gastric tumor after one course of chemotherapy. Of the 16 patients enrolled, 7 showed a partial response, 7 showed stable disease, and 1 had progressive disease, yielding an intent-to-treat ORR of $43.8 \%$. However, the response rate in the evaluable patients only was $46.7 \%$. Of the 7 patients with stable disease, 5 showed clinical improvement, such as an increase in appetite or a reduction in abdominal symptoms. For the 12 patients with a partial response or stable disease with clinical improvement, second-look laparoscopy was performed to assess the effectiveness of the chemotherapy against peritoneal metastasis. The metastases had disappeared in 4 of 10 patients with peritoneal dissemination, and peritoneal lavage cytology was negative in 5 of 6 patients whose results were previously positive (Fig. 2).

Table 1. Baseline characteristics of 16 patients

\begin{tabular}{lc}
\hline Sex & \\
Male & 12 \\
Female & 4 \\
Median age, years (range) & $66(48-73)$ \\
ECOG performance status & 8 \\
0 & 8 \\
1 & \\
Histological type & 1 \\
Intestinal & 15 \\
Diffuse & \\
Macroscopic type of primary tumor & 0 \\
1 & 2 \\
2 & 4 \\
3 & 10 \\
4
\end{tabular}

ECOG, Eastern Cooperative Oncology Group 
Table 2. Clinical courses of all patients

\begin{tabular}{|c|c|c|c|c|c|c|c|c|c|c|c|}
\hline \multirow{2}{*}{$\begin{array}{l}\text { Patient } \\
\text { no. }\end{array}$} & \multicolumn{2}{|c|}{$1^{\text {st }}$ Lap. } & \multirow{2}{*}{$\begin{array}{c}\text { Response to } \\
\text { treatment }\end{array}$} & \multicolumn{2}{|c|}{$2^{\text {nd }}$ Lap. } & \multirow{2}{*}{$\begin{array}{c}\text { Surgical } \\
\text { procedure }\end{array}$} & \multicolumn{2}{|c|}{ Intraoperative } & \multicolumn{3}{|c|}{ Final pathological findings } \\
\hline & $\mathrm{P}$ & $\mathrm{CY}$ & & $\mathrm{P}$ & $\mathrm{CY}$ & & $\mathrm{P}$ & $\mathrm{CY}$ & $\mathrm{T}$ & $\mathrm{N}$ & Stage \\
\hline 1 & 1 & 1 & PR & 1 & 0 & - & - & - & - & - & IV \\
\hline 2 & 1 & 1 & $\mathrm{SD} 2$ & - & - & - & - & - & - & - & IV \\
\hline 3 & 1 & 1 & PR & 0 & 0 & $\mathrm{~T}$ & 0 & 0 & 2 & 1 & II \\
\hline 4 & 0 & 1 & PR & 0 & 0 & $\mathrm{~T}$ & 0 & 1 & 3 & 2 & IV \\
\hline 5 & 1 & 0 & SD2 & - & - & - & - & - & - & - & IV \\
\hline 6 & 1 & 1 & SD1 & 1 & 0 & - & - & - & - & - & IV \\
\hline 7 & 1 & 1 & SD1 & 1 & 1 & - & - & - & - & - & IV \\
\hline 8 & 1 & 1 & $\mathrm{NE}$ & - & - & $(\mathrm{T})$ & 1 & - & 3 & 2 & IV \\
\hline 9 & 0 & 1 & PR & 0 & 0 & D & 0 & 0 & 2 & 3 & IV \\
\hline 10 & 1 & 0 & PR & 0 & 0 & $\mathrm{D}$ & 0 & 0 & 3 & 2 & IIIB \\
\hline 11 & 0 & 1 & SD1 & 0 & 0 & Bypass & 1 & 0 & 4 (panc) & $X$ & IV \\
\hline 12 & 0 & 1 & PR & 0 & 0 & D & 1 & 1 & 2 & 0 & IV \\
\hline 13 & 1 & 0 & SD1 & 0 & 0 & D & 0 & 0 & 2 & 2 & IIIA \\
\hline 14 & 0 & 1 & SD1 & 0 & 0 & $\mathrm{~T}$ & 0 & 0 & 3 & 2 & IIIB \\
\hline 15 & 1 & 1 & PR & 0 & 0 & $\mathrm{~T}$ & 0 & 0 & 2 & 0 & IB \\
\hline 16 & 0 & 1 & PD & - & - & - & - & - & - & - & IV \\
\hline
\end{tabular}

Response was evaluated by evaluable lesions, not by measurable lesions

Lap., laparotomy; P, peritoneal dissemination, CY, intra-abdominal lavage cytology; PR, partial response; SD1, stable disease with clinical benefit; $\mathrm{SD} 2$, stable disease without clinical benefit; $\mathrm{PD}$, progressive disease; NE, not evaluated; 0 , negative; 1 , positive; panc, pancreatic invasion Surgical procedure: T, total gastrectomy; (T), emergent total gastrectomy; D, distal gastrectomy; bypass, gastrojejunostomy

$1^{\text {st }}$ st-lap

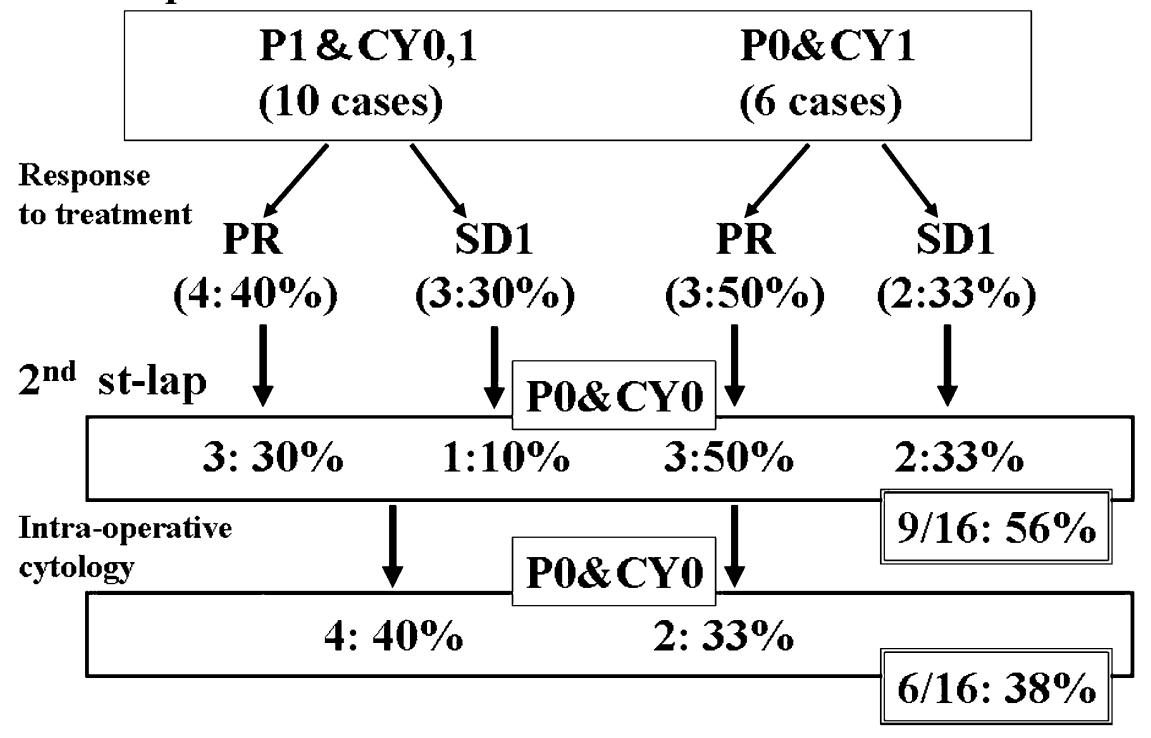

Fig. 2. Response to peritoneal lesions. Second-look laparoscopy was performed to assess effectiveness of the chemotherapy against peritoneal metastasis for 12 patients with a partial response or stable disease with clinical improvement. Metastases disappeared in 4 of 10 patients with peritoneal dissemination, and peritoneal lavage cytology became negative in 5 of 6 patients who were previously positive. Disappearance of peritoneal dissemination was diagnosed in 4 of 10 patients, and negative peritoneal lavage cytology was diagnosed in 2 of 6 patients who had been positive at the time of surgery. Disappearance of peritoneal dissemination was eventually confirmed in $37.5 \%$ of the patients (6/16 cases). st-lap, Staging laparoscopy; $S D 1$, stable disease with clinical benefit

\section{Adverse reactions}

All patients (except for the one who received only one course of chemotherapy because of emergent gastrectomy) were evaluated for toxic effects. The incidences of the main adverse reactions, scored as the highest grade seen per patient during this chemotherapy, are listed in Table 3. Grade 3 leukopenia was observed in $13.3 \%(2 / 15)$ of the patients, and grade 3 neutropenia in $20.0 \%(3 / 15)$. The incidences of AST, ALT, and bilirubin increases were $20.0 \%$, $20.0 \%$, and $13.3 \%$, respectively. However, except for leucopenia and neutropenia, there was no hematological toxicity of grade 3 or higher in any patient. Alopecia was the most frequent adverse reaction, with an incidence of $100 \%$. Nonhematological toxicities of grade 3 or higher were not observed in any patient (Table $3)$.

There was no treatment-related death or delayed severe toxicity. 
Table 3. Adverse reactions during chemotherapy expressed by grade $(n=15)$

\begin{tabular}{lrrrrr}
\hline & \multicolumn{5}{c}{ Grade } \\
\cline { 2 - 6 } & 1 & 2 & 3 & 4 & Incidence (\%) \\
& & & & & \\
Hematological & 3 & 2 & 2 & 0 & 46.7 \\
$\quad$ Leucopenia & 1 & 1 & 3 & 0 & 33.3 \\
Neutropenia & 10 & 2 & 0 & 0 & 80.0 \\
Anemia & 2 & 0 & 0 & 0 & 13.3 \\
Thrombocytopenia & & & & & \\
Laboratory values & 3 & 0 & 0 & 0 & 20.0 \\
AST & 3 & 0 & 0 & 0 & 20.0 \\
ALT & 0 & 2 & 0 & 0 & 13.3 \\
Bilirubin & 0 & 0 & 0 & 0 & 0 \\
BUN & 0 & 0 & 0 & 0 & 0 \\
Creatinine & & & & & \\
Nonhematological & 9 & 1 & 0 & 0 & 66.6 \\
Fatigue & 8 & 1 & 0 & 0 & 60.0 \\
Anorexia & 1 & 0 & 0 & 0 & 6.7 \\
Stomatitis & 1 & 0 & 0 & 0 & 6.7 \\
Nausea/vomiting & 2 & 0 & 0 & 0 & 13.3 \\
Diarrhea & 4 & 12 & & & 100 \\
Alopecia &
\end{tabular}

Numbers in columns are numbers of patients

AST, aspartate aminotransferase; ALT, alanine aminotransferase; BUN, blood urea nitrogen

\section{Surgical treatment and prognosis}

We performed elective surgery in 9 patients in whom peritoneal dissemination had disappeared and/or lavage cytology had become negative on second-look laparoscopy.

The procedures performed are listed in Table 2. Four patients underwent total gastrectomy with lymph node dissection and four underwent distal gastrectomy, and one underwent gastrojejunostomy because of invasion of the pancreatic head. Apart from these patients, one patient received an emergency total gastrectomy for bleeding.

At the time of surgery, disappearance of peritoneal dissemination was diagnosed in 4 of 10 patients by based on several histological examinations (e.g., presence or absence of white scar on the peritoneum), and negative peritoneal lavage cytology was diagnosed in 2 of 6 patients who had been positive at the time of surgery. The disappearance of peritoneal metastasis was eventually confirmed in $37.5 \%$ of the patients $(6 / 16$ cases: 95\% confidence interval [CI], 13.0-62.0; Fig. 2).

Pathological examination of the resected specimen demonstrated that grade 2 tumor regression was achieved in two patients, grade $1 \mathrm{~b}$ in one, and grade $1 \mathrm{a}$ in five. On the final pathological examination of the resected specimens, one patient was stage IB, one was stage II, three were stage III, and the remaining four were stage IV. in the final pathological examination of resected specimens, In addition to these patients, there

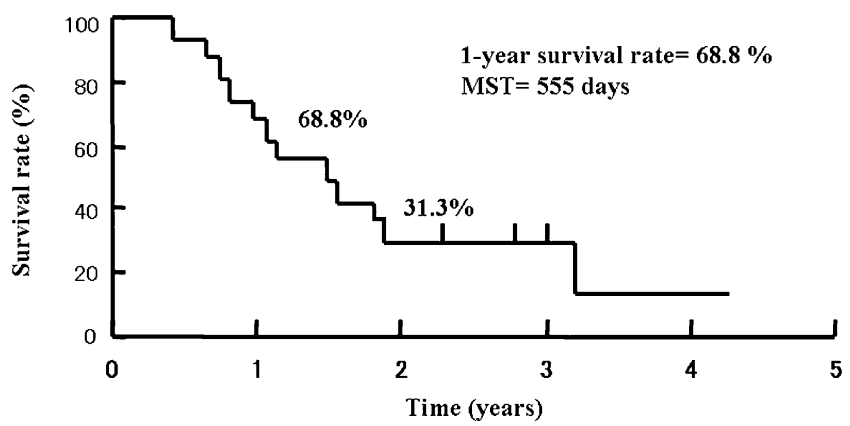

Fig. 3. Cumulative probability of overall survival estimated by the Kaplan-Meier method for 16 patients. $M S T$, median survival time

were 7 patients including a case with gastrojejunostomy in stage IV whose primary tumor was not resected (Table 2).

There were no postoperative complications in any patient.

The 1- and 2-year survival rates were $68.8 \%$ and $31.3 \%$, respectively, and the MST was 555 days (95\% CI, 233-819 days; Fig. 3).

\section{Discussion}

Although peritoneal dissemination commonly occurs in advanced gastric cancer, it is difficult to diagnose preoperatively by routine examinations such as abdominal ultrasonography and CT, except in patients with ascites. Laparoscopy was reported to be an important diagnostic tool for the staging of advanced gastric cancer [20, 21], and we too have performed it for patients with clinical T3 gastric cancer, by preoperative multidetector row CT since September 2002. Although staging laparoscopy facilitates a more accurate preoperative diagnosis of peritoneal dissemination, there is no standard treatment for peritoneal dissemination.

$\mathrm{S}-1$, developed as an oral drug for the treatment of gastric cancer, has been reported to be effective against advanced and recurrent gastric cancer, including cases of peritoneal dissemination [4-7]. S-1 has been reported to be more effective than conventional systemic chemotherapy against peritoneal dissemination, because high concentrations of 5-fluorouracil (5-FU) are selectively transferred to sites of dissemination. This occurs because $\mathrm{S}-1$ contains gimeracil [CDHP; an inhibitor of 5-FU metabolic enzymes dihydropyrimidine dehydrogenase (DPD)], and CHDF passes through the peritoneum and disseminated nodes with 5-FU after oral administration of S-1, inhibiting its metabolism of 5-FU, and this inhibition plays an important role in the delivery of 5-FU to peritoneal disseminated cells [22]. Recently, Hosokawa et al. [23] reported that the median survival time of 
patients with only peritoneal metastasis was 24 months following initial S-1 monotherapy.

While S-1 was more effective than conventional chemotherapy against peritoneal dissemination, when it was combined with other anticancer drugs such as cisplatin (CDDP), taxanes, and CPT-11, S-1 increased ORRs and prolonged MST [9-11].

The results of the SPIRITS study (S-1 vs S-1+CDDP) proved the superiority of the S-1+CDDP combination over S-1 monotherapy [24]. The rate of response to the combination therapy versus monotherapy was 54\% versus $31 \%(P=0.0018)$, and the MST was 13.0 versus 11.0 months $(P=0.0366)$. Moreover, a subgroup analysis suggested that the presence of peritoneal metastasis was an indication for chemotherapy with S-1 plus CDDP, although the efficacy for peritoneal dissemination itself has remained unclear.

For patients with peritoneal dissemination, systemic chemotherapy with methotrexate (MTX)/5-FU or intraperitoneal chemotherapy has been performed.

Sequential MTX/5-FU therapy was effective in a phase II trial for advanced gastric cancer [25, 26]. In a Japanese phase II trial of sequential MTX/5-FU for patients with advanced gastric cancer, the response rates were reported to be $23 \%$ and $41 \%$ with low- and intermediate-dose MTX regimens, respectively [27]. Konishi et al. [28] reported that the MTX/5-FU regimen showed greater efficacy in patients with the diffuse type of adenocarcinoma than in those with the intestinal type. The response rate of patients with peritoneal dissemination in their study was $23 \%(6 / 26)$ and ascites was eliminated in 8 of 16 patients (50\%). Tahara et al. [29] reported that sequential MTX/5-FU therapy for patients with confirmed peritoneal dissemination was effective, and an objective attenuation of ascites was seen in 13 of 26 patients, including 5 who showed complete disappearance of ascites; the MST and median time to treatment failure were 259 days and 167 days, respectively.

Moreover, in a multicenter phase II trial of sequential MTX/5-FU therapy, the response rate of ascites was $35.1 \%$, although the overall objective response rate was $5.7 \%$ [30]. Although sequential MTX/5-FU therapy was effective against advanced gastric cancer with malignant ascites, its overall results have been unsatisfactory.

Recently, some investigators have reported good outcomes with intraperitoneal chemotherapy for peritoneal dissemination [31,32]. These studies demonstrated that intraperitoneal chemotherapy could deliver a high dose into the peritoneal cavity. As taxanes have a high molecular weight and bulky chemical structure, resulting in a delay in peritoneal clearance and an increase in the time they remain in the peritoneal cavity [33], they can be exploited in the treatment of gastric cancer with peritoneal dissemination.
We performed a pilot study of combined chemotherapy with both intraperitoneal paclitaxel and oral S-1 for patients with peritoneal metastasis and obtained a good result [34].

We submitted this protocol to the institutional review board of our hospital for a phase II study in 2003, but were rejected because of several problems concerning expansion of the clinical utilization of intraperitoneal paclitaxel administration in Japan. Therefore, we started the intravenous administration of paclitaxel and oral S-1 as a new trial using the same dose intensity as that used for the intraperitoneal chemotherapy [34].

Paclitaxel was administered as a single agent every 3 weeks at a dose of $210 \mathrm{mg} / \mathrm{m}^{2}$ in two phase II trials performed in Japan [35, 36]. Subsequently, however, paclitaxel came to be widely used in a weekly schedule of $80 \mathrm{mg} / \mathrm{m}^{2}$ in Japan [37, 38], referring to reports that bone marrow toxicity was mild and manageable when compared to the triweekly schedule, although the weekly paclitaxel was not superior to the triweekly regimen in terms of ORRs. In combinations with S-1, paclitaxel was widely used at a dose of 50 or $60 \mathrm{mg} / \mathrm{m}^{2}$ in a weekly setting $[10,14,39]$, but the weekly schedule had not yet been approved in Japan at the time that we carried out the present study. Therefore, we selected the triweekly schedule for the administration of paclitaxel. According to the dosage of paclitaxel, this drug was administered weekly at a dose of 50 or $60 \mathrm{mg} / \mathrm{m}^{2}$ two or three times every 3 or 4 weeks. Therefore, dose intensity per week was calculated as ranging from 33.3 to $45.0 \mathrm{mg} /$ $\mathrm{m}^{2}$. Although we adopted $120 \mathrm{mg} / \mathrm{m}^{2}$ of paclitaxel to match the dose to that used for intraperitoneal administration in our previous study [34], our dose intensity of $40 \mathrm{mg} / \mathrm{m}^{2}$ per week seemed to be sufficiently high, suggesting that the combination of $\mathrm{S}-1+$ paclitaxel resulted in a favorable response rate and MST.

In the present study, the response rate and MST were $44 \%$ and 555 days, respectively, comparable with those in the SPIRITS trial [24] and other paclitaxel plus S-1 studies [10,14], although differences in response rate preclude direct comparisons because we did not evaluate the response by RECIST.

As patients with measureable metastatic lesions were excluded from the present study, the tumor burden in our study patients was relatively small compared to those in other clinical trials for advanced gastric cancer, including the SPIRITS trial [24]. However, the prognosis in patients with peritoneal dissemination has been reported to be poor regardless of the tumor burden, even in patients with only positive peritoneal lavage cytology $[15,16]$. More recently, in some new clinical trials for patients with advanced gastric cancer with macroscopic peritoneal metastasis and/or positive peritoneal cytology without any other distant metastasis, there were some patients with dramatically prolonged 
survival following the administration of S-1 or other treatments such as intraperitoneal administration of taxanes or intraoperative peritoneal hyperthermic chemotherapy after surgical resection of the primary tumor $[32,34,40,41]$. These new clinical trials may be able to offer treatment options to patients with peritoneal metastasis.

In the present study, we found that the combination of S-1 and triweekly paclitaxel was feasible even in patients receiving surgical resection. Moreover, the complete response rate for peritoneal metastasis was $37.5 \%$ by cytopathological examination both at secondlook laparoscopy and surgery, and radical resections were performed for these five patients who achieved a downstaging of gastric cancer. As it is difficult to judge the effectiveness of chemotherapy for peritoneal metastasis solely by routine inspections, it seems that our rate of $37.5 \%$ for the disappearance of peritoneal disease may become a reference value.

To obtain clear evidence about first-line chemotherapy for patients with peritoneal metastasis, a randomized clinical trial comparing S-1 plus CDDP and S-1 plus i.v. or i.p. administration of other anticancer agents such as taxanes, is necessary. Moreover, it will be necessary to examine whether or not surgical resection of the primary gastric tumor contributes to the prolongation of survival. Recently, Kodera et al. [37] reported a phase II study of radical surgery followed by postoperative chemotherapy with S-1 for gastric carcinoma with free cancer cells in the peritoneal cavity, in which the MST was 705 days and the 2-year survival rate was $47 \%$. These good results, unlike past results, suggest that, for patients with peritoneal dissemination, radical surgery followed by effective treatment may contribute to an improvement of survival $[15,16]$.

The chemotherapy regimen used in the present study is thought to be effective and feasible for patients with peritoneal metastasis from gastric cancer. Because this was a small pilot study, we have planned a phase II study to further clarify the effects and safety of this combination chemotherapy for gastric cancer patients with peritoneal dissemination.

Acknowledgments We express our appreciation to Dr. Shinichi Nakatsuka, Department of Pathology at our institute, for his expert comments.

\section{References}

1. Kakeji Y, Maehara Y, Tomoda M, Kabashima A, Ohmori M, Oda $\mathrm{S}$, et al. Long term survival of patients with stage IV gastric cancer. Cancer 1998;82:2307-11.

2. Yonemura Y, Sawa T, Kinoshita K, Matsuki N, Fushida S, Tanaka $\mathrm{S}$, et al. Neoadjuvant chemotherapy for high-grade advanced gastric cancer. World J Surg 1993;17:256-62.
3. Hagiwara A, Takahashi T, Kojima O, Sawai K, Yamaguchi T, Yamane T, et al. Prophylaxis with carbon-absorbed mitomycin C against peritoneal recurrence of gastric cancer. Lancet 1992;339: 629-31.

4. Sakata Y, Ohtsu A, Horikoshi N, Sugimachi K, Mitachi Y, Taguchi T. Late phase II study on novel oral fluoropyrimidine anticancer drug S-1 (IM Tegafur-0.4M Gimestat-1M Otastat Potassium) in advanced gastric cancer patients. Eur J Cancer 1998;34:1715-20.

5. Koizumi W, Kurihara M, Nakano S, Hasegawa K. Phase II study of S-1, a novel oral derivative of 5-fluorouracil, in advanced gastric cancer. Oncology 2000;58:191-7.

6. Osugi H, Takada N, Takemura M, Kaseno S, Lee S, Ueno M, et al. Oral fluoropyrimidine anticancer drug TS-1 for gastric cancer patients with peritoneal dissemination. Oncol Rep 2002;9:8115 .

7. Kitamura Y, Hayashi K, Sasagawa T, Oguma H, Takasaki K. Pilot study of S-1 in patients with disseminated gastric cancer. Drugs Exp Clin Res 2003;29:125-30.

8. Koizumi W, Tanabe S, Saigenji T, Ohtsu A, Boku N, Nagashima F, et al. Phase I/II study of S-1 combined with cisplatin in patients with advanced gastric cancer. Br J Cancer 2003;89:2207-12.

9. Yoshida K, Ninomiya M, Takakura N, Hirabayashi N, Takiyama W, Sato Y, et al. Phase II study of docetaxel and S-1 combination therapy for advanced or recurrent gastric cancer. Clin Cancer Res 2006;12:3402-7.

10. Mochiki E, Ohno T, Kamiyama Y, Aihara R, Haga N, Ojima H, et al. Phase I/II study of S-1 combined with paclitaxel in patients with unresectable and/or recurrent advanced gastric cancer. Br J Cancer 2006;95:1642-7.

11. Inokuchi M, Yamashita T, Yamada H, Kojima K, Ichikawa W, Nihei Z, et al. Phase I/II study of S-1 combined with irinotecan for metastatic advanced gastric cancer. $\mathrm{Br}$ J Cancer 2006;94: $1130-5$.

12. Rowinsky EK, Donehower RC, Jones RJ, Tucker RW. Microtubule changes and cytotoxicity in leukemic cell lines treated with taxol. Cancer Res 1988;48:4093-100.

13. Kobayashi M, Sakamoto J, Namikawa T, Okamoto K, Okabayashi $\mathrm{T}$, Ichikawa K, et al. Pharmacokinetic study of paclitaxel in malignant ascites from advanced gastric cancer patients. World J Gastroenterol 2006;12:1412-5.

14. Narahara H, Fujitani K, Takiuchi H, Sugimoto N, Inoue K, Uedo $\mathrm{N}$, et al. Phase II study of a combination of S-1 and paclitaxel in patients with unresectable or metastatic gastric cancer. Oncology 2008;74:37-41.

15. Kodera Y, Yamamura Y, Shimizu Y, Torii A, Hirai T, Yasui K, et al. Peritoneal wash cytology: prognostic value of positive findings in patients with gastric carcinoma undergoing a potentially curative resection. J Surg Oncol 1999;72:60-5.

16. Burke EC, Karpeh MS Jr, Conlon KC, Brennan MF. Peritoneal lavage cytology in gastric cancer: an independent predictor of outcome. Ann Surg Oncol 1998;5:411-5.

17. Tamura S, Miki H, Nakata K, Takiuchi D, Okada K, Nakahira S, et al. Intraperitoneal administration of paclitaxel and oral S-1 for patients with peritoneal dissemination and hydronephrosis due to advanced gastric cancer. Gastric Cancer 2007;10:251-5.

18. Japanese Gastric Cancer Association. Japanese classification of gastric carcinoma. - 2nd English Edition -. Gastric Cancer 1998;1:10-24.

19. Japanese Gastric Cancer Association. Japanese classification of gastric carcinoma. - 2nd English Edition - Response assessment of chemotherapy and radiotherapy for gastric carcinoma: clinical criteria. Gastric Cancer 2001;4:1-8.

20. Sotiropoulos GC, Kaiser GM, Lang H, Treckmann J, Brokalaki EI, Pottgen C, et al. Staging laparoscopy in gastric cancer. Eur J Med Res 2005;10:88-91.

21. Nakagawa S, Nashimoto A, Yabusaki H. Role of staging laparoscopy with peritoneal lavage cytology in the treatment of locally advanced gastric cancer. Gastric Cancer 2007;10:29-34. 
22. Oshima T, Yamada R, Hatori S, Kunisaki C, Imada T. Pharmacokinetics of S-1 in patients with peritoneal dissemination of gastric cancer. Oncol Rep 2006;16:361-6.

23. Hosokawa A, Sugiyama T, Ohtsu A, Doi T, Hattori S, Kojima T, et al. Long-term outcomes of patients with metastatic gastric cancer after initial S-1 monotherapy. J Gastroenterol 2007;42: 533-8.

24. Koizumi W, Narahara H, Hara T, Takagane A, Akiya T, Takagi M, et al. S-1 plus cisplatin versus S-1 alone for first-line treatment of advanced gastric cancer (SPIRITS trial): a phase III trial. Lancet Oncol 2008;9:215-21.

25. Dickinson R, Presgrave P, Levi J, Milliken S, Woods R. Sequential moderate-dose methotrexate and 5-fluorouracil in advanced gastric adenocarcinoma. Cancer Chemother Pharmacol 1989;24: 67-8.

26. Pérez JE, Lacava JA, Dominguez ME, Rodriguez R, Barbieri MR, Ortiz EH, et al. Biochemical modulation of 5-fluorouracil by methotrexate in patients with advanced gastric carcinoma. Am J Clin Oncol 1998;21:452-7.

27. Murakami M, Ota K, Miyazaki T, Niitsu Y, Wakui A, Yokoyama $\mathrm{M}$, et al. Sequential methotrexate-5-fluorouracil (MTX-5-FU) treatment for patients with advanced gastric and colorectal cancer (in Japanese with English abstract). Gan To Kagaku Ryoho 1987;14:2482-90.

28. Konishi T, Hiraishi M, Mafune K, Miyama T, Hirata T, Mori K, et al. Therapeutic efficacy and toxicity of sequential methotrexate and 5-fluorouracil in gastric cancer. Anticancer Res 1994;14: 1277-80.

29. Tahara M, Ohtsu A, Boku N, Nagashima F, Muto M, Sano Y, et al. Sequential methotrexate and 5-fluorouracil therapy for gastric cancer patients with peritoneal dissemination: a retrospective study. Gastric Cancer 2001;4:212-8.

30. Yamao T, Shimada Y, Shirao K, Ohtsu A, Ikeda N, Hyodo I, et al. Phase II study of sequential methotrexate and 5-fluorouracil chemotherapy against peritoneally disseminated gastric cancer with malignant ascites: a report from the Gastrointestinal Oncology Study Group of the Japan Clinical Oncology Group, JCOG 9603 Trial. Jpn J Clin Oncol 2004;34:316-22.

31. Fushida S, Furui N, Kinami S, Ninomiya I, Fujimura T, Nishimura $\mathrm{G}$, et al. Pharmacologic study of intraperitoneal paclitaxel in gastric cancer patients with peritoneal dissemination (in Japanese with English abstract). Gan To Kagaku Ryoho 2002;29:2164-7.
32. Yonemura Y, Endou Y, Bando E, Kuno K, Kawamura T, Kimura $\mathrm{M}$, et al. Effect of intraperitoneal administration of docetaxel on peritoneal dissemination of gastric cancer. Cancer Lett 2004;210: 189-96.

33. Markman M, Rowinsky E, Harkes T, Reichman B, Jones W, Lewis JL Jr, et al. Phase I trial of intraperitoneal Taxol: a Gynecologic Oncology Group Study. J Clin Oncol 1992;10:1485-91.

34. Tamura S, Miki H, Okada K, Miyake T, Yoshimura M, Suzuki R, et al. Pilot study of intraperitoneal administration of paclitaxel and oral S-1 for patients with peritoneal metastasis due to advanced gastric cancer. Int J Clin Oncol 2008;13:536-40.

35. Yamada Y, Shirao K, Ohtsu A, Boku N, Hyodo I, Saitoh H, et al. Phase II trial of paclitaxel by 3-hour infusion for advanced gastric cancer with short premedication for prophylaxis against paclitaxel-associated hypersensitivity reaction. Ann Oncol 2001; 12:1133-7.

36. Yamaguchi K, Tada M, Horikoshi N, Otani T, Takiuchi H, Saitoh $\mathrm{S}$, et al. Paclitaxel Gastric Cancer Study Group in Japan, phase II study of paclitaxel with 3 -h infusion in patients with advanced gastric cancer. Gastric Cancer 2002;5:90-5.

37. Kodera Y, Ito S, Mochizuki Y, Fujikake S, Koshikawa K, Kanyama Y, et al. Chubu Clinical Cancer Group. A phase II study of weekly paclitaxel as second-line chemotherapy for advanced gastric cancer (CCOG0302 study). Anticancer Res 2007;27:2667-71.

38. Emi Y, Yamamoto M, Takahashi I, Orita H, Kakeji Y, Kohnoe S, et al. Phase II study of weekly paclitaxel by 1-hour infusion for advanced gastric cancer. Surg Today 2008;38:1013-20.

39. Ueda Y, Yamagishi H, Ichikawa D, Mori J, Koizumi K, Kakihara $\mathrm{N}$, et al. Phase I study of a combination of S-1 and weekly paclitaxel in patients with advanced or recurrent gastric cancer. Oncology 2005;69:261-8.

40. Li C, Yan M, Chen J, Xiang M, Zhu ZG, Yin HR, et al. Survival benefit of non-curative gastrectomy for gastric cancer patients with synchronous distant metastasis. J Gastrointest Surg 2010; 14:282-8.

41. Kodera Y, Ito S, Mochizuki Y, Kondo K. Koshikawa K, Suzuki N, et al. A phase II study of radical surgery followed by postoperative chemotherapy with S-1 for gastric carcinoma with free cancer cells in the peritoneal cavity (CCOG0301 study). Eur J Sur Oncol 2009;35:1158-63. 